\title{
Magnetic Hyperthermia Experiments with Magnetic Nanoparticles in Clarified Butter Oil and Paraffin: A Thermodynamic Analysis
}

\author{
Adriele A. de Almeida, Emilio De Biasi, Marcelo Vasquez Mansilla, Daniela P. Valdés, Horacio E. Troiani, \\ Guillermina Urretavizcaya, Teobaldo E. Torres, Luis M. Rodríguez, Daniel E. Fregenal, \\ Guillermo C. Bernardi, Elin L. Winkler, Gerardo F. Goya, Roberto D. Zysler, and Enio Lima, Jr.*
}

Cite This: https://dx.doi.org/10.1021/acs.jpcc.0c06843

ABSTRACT: In specific power absorption models for magnetic fluid hyperthermia (MFH) experiments, the magnetic relaxation time of nanoparticles (NPs) is known to be a fundamental descriptor of the heating mechanisms. The relaxation time is mainly determined by the interplay between the magnetic properties of NPs and the rheological properties of NPs' environment. Although the role of magnetism in $\mathrm{MFH}$ has been extensively studied, the thermal properties of the NP medium and their changes during MFH experiments have been underrated so far. Herein, we show that $\mathrm{Zn}_{x} \mathrm{Fe}_{3-x} \mathrm{O}_{4}$ NPs dispersed through different media with phase transition in the temperature range of experiment as clarified butter oil (CBO) and paraffin. These
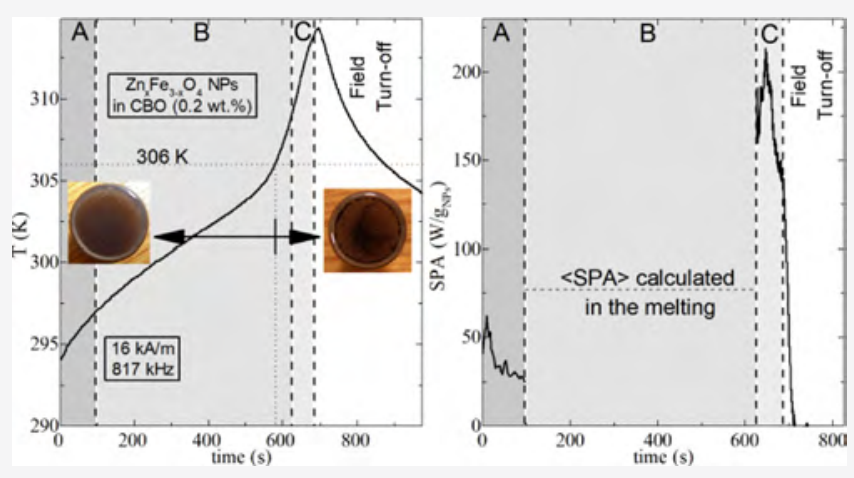
systems show nonlinear behavior of the heating rate within the temperature range of MFH experiments. For CBO, a fast increase at $\sim 306 \mathrm{~K}$ is associated with changes in the viscosity $(\eta(T))$ and specific heat $\left(c_{p}(T)\right)$ of the medium at its melting temperature. This increment in the heating rate takes place around $318 \mathrm{~K}$ for paraffin. The magnetic and morphological characterization of NPs together with the observed agglomeration of NPs above 306 and $318 \mathrm{~K}$ for $\mathrm{CBO}$ and paraffin, respectively, indicate that the fast increase in MFH curves could not be associated with the change in the magnetic relaxation mechanism, with Néel relaxation being dominant. In fact, successive experimental runs performed up to temperatures below and above the $\mathrm{CBO}$ and paraffin melting points resulted in different MFH curves due to agglomeration of NPs driven by magnetic field inhomogeneity during the experiments. Our results highlight the relevance of the thermodynamic properties of the system NP-medium for an accurate measurement of the heating efficiency for in vitro and in vivo environments, where the thermal properties are largely variable within the temperature window of MFH experiments.

\section{INTRODUCTION}

In a magnetic fluid hyperthermia (MFH) experiment, the relaxation time of the magnetic moment $(\tau)$ of nanoparticles (NPs) is a key factor for heating mechanisms operating behind specific power absorption (SPA) determination. In a single domain NP, effective heating results from the interplay of two relaxation mechanisms: the Brownian rotation against the viscous forces expressed by $\tau_{\mathrm{B}}=3 \eta V_{\text {hyd }} / k_{\mathrm{B}} T$, where $\eta$ is the viscosity of the medium, $V_{\text {hyd }}$ is the hydrodynamic volume of the NP, and $k_{\mathrm{B}} T$ is the thermal energy, and the Neel relaxation of the magnetic moment expressed by $\tau_{\mathrm{N}}=\tau_{0} \exp \left(K_{\mathrm{eff}} V / k_{\mathrm{B}} T\right)$, where $\tau_{0}$ is the characteristic attempt time and $K_{\text {eff }} V$ is the effective anisotropy energy barrier of the NP given by its effective anisotropy constant $K_{\text {eff }}$ and volume $V$. ${ }^{1}$ The dominant mechanism will be the one with a shorter relaxation time. For MFH applications, the most adequate systems are those in which $\tau_{\mathrm{N}}$ dominates ${ }^{2-5}$ because the heating power depends only on the intrinsic magnetic properties of NPs, and it is independent of the (variable) viscosity of different biological media. Therefore, the precise heat dose delivered is independent of the specific tissue or organ targeted. ${ }^{6-10}$ There is a growing consensus on the fact that magnetic NPs for MFH should be fabricated in this way, and optimization is done by adjusting its $K_{\text {eff }} V$.

Only a few studies have been reported so far, evaluating the variability of SPA expected when the NPs are dispersed in a medium with a viscosity $[\eta(T)]$ and specific heat $\left[c_{p}(T)\right]$, which are temperature-dependent within the temperature range measured. ${ }^{11}$ If the medium presents a strong reduction of $\eta(T)$ in the temperature range of the experiment, for instance, due to melting, it results in a shorter $\tau_{\mathrm{B}}$, which may

Received: July 26, 2020

Revised: November 14, 2020 
lead to a change in the dominant mechanism acting behind the heating process. However, even when the magnetic relaxation $\left(\tau_{\mathrm{N}}\right)$ is still dominating, changes in the properties of medium may affect the SPA measured for the system. Changes in viscosity may affect the spatial arrangement of the NPs, allowing the alignment of their magnetic easy axis with the external field gradient and yielding the formation of multiparticle structures with a very different SPA response. ${ }^{12-14}$ In the case of phase transition such as melting, the latent heat $(L)$ also impacts on the SPA measured.

Paraffin and clarified butter oil (CBO) are both materials that fit our needs for the present study on thermal evolution of the SPA parameter during MFH experiments. Both substances show a phase transition at temperatures within the $\mathrm{MFH}$ experimental range. Paraffin is a uncomplicated medium in terms of composition, made of a mixture of linear $n$-alkanes $\left(\mathrm{CH}_{3}-\left(\mathrm{CH}_{2}\right)_{n}-\mathrm{CH}_{3}\right)$ with a phase transition that depends on the number of carbon atoms involved (e.g., $293 \mathrm{~K}<T<295 \mathrm{~K}$ for $\mathrm{C}_{16}-\mathrm{C}_{18}$ and $339 \mathrm{~K}<\mathrm{T}<341 \mathrm{~K}$ for $\left.\mathrm{C}_{21}-\mathrm{C}_{50}\right) .{ }^{15}$ It has a latent heat $L$ of $121.9 \mathrm{~J} / \mathrm{g}$, a thermal conductivity of $\sim 0.21-$ $0.24 \mathrm{~W} / \mathrm{mK}$ just below the melting point, ${ }^{15}$ and a dynamic viscosity around $\eta \sim 2.1 \mathrm{mPa} \cdot \mathrm{s}$ at the melting temperature of $T_{\mathrm{M}}=332 \mathrm{~K} \cdot{ }^{16} \mathrm{CBO}$ is a more complex medium obtained by extraction from heated butter oil, which could be interesting when considering how to emulate the potential of NPs candidates for MFH applications. It is composed of 99-99.5\% of fatty acids-palmitic, oleic, myristic, and stearic acid. ${ }^{17}$ The use of $\mathrm{CBO}$ avoids the presence of water droplets that can affect the solidification process of the fat, and consequently, the physicochemical properties of the crystal network. ${ }^{18}$ Physical properties of $\mathrm{CBO}$ are well known, with $T_{\mathrm{M}}=305.2 \mathrm{~K}^{19}$ (compared to $T_{\mathrm{M}}=308.0 \mathrm{~K}$ reported for butter $^{20}$ ). The viscosity of $\mathrm{CBO}$ decreases at the melting point by a factor of $10^{3}$, from some $\mathrm{Pa} \cdot \mathrm{s}$ to tens of $\mathrm{mPa} \cdot \mathrm{s}^{21}$ Also, thermal properties, such as $c_{p}$, vary with temperature, being sensitive to changes in its physical state close to $T_{\mathrm{M}}{ }^{22}$ In fact, there are distinct regions of phase transition related to melting: the lowmelting fraction (LMF), around $280 \mathrm{~K}$, medium-melting fraction (MMF), around $290 \mathrm{~K}$, and a high-melting fraction (HMF), around $310 \mathrm{~K}$, where the temperature range depends on the heating rate. ${ }^{23}$ Its thermal conductivity slightly before the melting point is estimated at $0.22 \mathrm{~W} / \mathrm{m} \cdot \mathrm{K}^{21}$

In this work, we study the SPA of $\mathrm{Zn}_{x} \mathrm{Fe}_{3-x} \mathrm{O}_{4}$ NPs dispersed in $\mathrm{CBO}$ and paraffin, two media with a strong reduction of viscosity at the phase transition within the temperature range of MFH experiments. In particular, we analyze NPs with different shapes, sizes, and initial heating rates dispersed in these two media, and the effects of particle agglomeration, observed at higher temperatures, on the SPA. For this, we synthesized $\mathrm{Zn}_{x} \mathrm{Fe}_{3-x} \mathrm{O}_{4}$ NPs with different mean diameters $(\langle d\rangle)$ and dispersions $(\sigma)$, as well as different magnetic anisotropy constants $\left(K_{\text {eff }}\right)$. The variations in $K_{\text {eff }}\langle d\rangle$, and $\sigma$ of NPs result in different measured heating rates, and consequently, to different values of SPA. The incorporation of $\mathrm{Zn}$ in the ferrite structure reduces the anisotropy of the system, allowing the heating up to lower temperatures, below the transition temperature of the medium, so within the highviscosity range. A nonusual thermal variation of the heating rate is observed in MFH experiments, assigned to changes in the properties of the medium with temperature, together with a spatial rearrangement of the NPs. Finally, irreversibility in the MFH response after successive experiments (runs) is observed for samples with higher NP concentrations, resulting from agglomeration above the melting point of $\mathrm{CBO}$ and paraffin.

\section{METHODS}

$\mathrm{Zn}_{x} \mathrm{Fe}_{3-x} \mathrm{O}_{4}$ NPs were produced by the thermal decomposition at high temperatures of $\mathrm{Fe}(\mathrm{III})$ and $\mathrm{Zn}(\mathrm{II})$ acetylacetonate in different solvents (benzyl ether and trioctylamine) using the surfactants oleic acid and oleylamine. The method was adapted from the procedure described by Lohr et al., ${ }^{24}$ trying to avoid the presence of Wüstite phase. The composition of each sample, with general formula $\mathrm{Zn}_{x} \mathrm{Fe}_{3-x} \mathrm{O}_{4}$ with $x$ varying in the $0.1-0.2$ range, was controlled by the precursor ratio used. The as-prepared NPs are hydrophobic, coated with oleic acid. In order to understand the dominant relaxation mechanisms in MFH experiments, we performed a morphological characterization and correlated the observed magnetic properties with the phase composition of NPs.

The composition of the as-prepared NPs was determined by particle-induced X-ray emission (PIXE) ${ }^{25}$ using a $3 \mathrm{MeV} \mathrm{H}^{+}$ beam from a NEC 5SDH 1.7 MV tandem accelerator with a NEC RC43 end-station. For this, NPs were washed several times with acetone in order to reduce the oleic acid and oleylamine amounts remaining from the synthesis and dried. The resulting powder was conditioned over a carbon tape for PIXE measurements, and the results were analyzed with software GUPIX. ${ }^{26}$ As the PIXE technique is very sensitive, the following signals from other elements, arising from sample handling and conditioning, are observed: carbon, aluminum, sodium, calcium, and silicon. Therefore, the composition of each sample was focused only in determining the ratio between zinc and iron amounts $([\mathrm{Zn}]$ and $[\mathrm{Fe}]$, respectively), resulting in $x=0.11,0.12,0.19$, and 0.21 (see Figure S1 in pg. S1 of Supporting Information). Samples were labeled according to this.

The size and diameter dispersion of as-prepared NPs were determined by transmission electron microscopy (TEM) performed on a Philips CM200 microscope operating at 200 $\mathrm{kV}$. TEM specimens were prepared by dropping a solution of NPs dispersed in toluene onto a copper grid coated with an amorphous carbon layer. The diameter histograms were built up by measuring the diameter of at least $300 \mathrm{NPs}$, and the mean diameter $\langle d\rangle$ and dispersion $\sigma$ were obtained by fitting them with a lognormal distribution. A typical XRD profile (sample $x=0.21$ ) of samples is shown in Figure S2, pg. S2 of Supporting Information. All diffraction peaks observed could be indexed by a cubic magnetite phase (JCPDS card 19-0629). No evidence of $\mathrm{ZnO}$ phase was observed within the limit of our background and peak width, as estimated from the absence of most intense peaks of the hexagonal $\mathrm{ZnO}$ phase (JCPDS card 36-1451). Thus, we assumed that the $\mathrm{Zn}$ contents obtained from PIXE analysis were incorporated into the cubic ferrite structure.

The magnetic properties of systems were measured using a vibrating sample magnetometer (VSM, Lakeshore) and in a commercial superconducting quantum interference device magnetometer (SQUID, MPMS Quantum Design). The saturation magnetization $\left(M_{\mathrm{S}}\right)$ was determined by magnetization measurements as a function of the applied field measured at $300 \mathrm{~K}$ using the VSM (see Figure S3 in pg. S3 of Supporting Information). The blocking temperature distribution $\left(f\left(T_{\mathrm{B}}\right)\right)$ of each sample was obtained from the magnetization curves as a function of temperature measured in the zero-field-cooling (ZFC) and field-cooling (FC) 

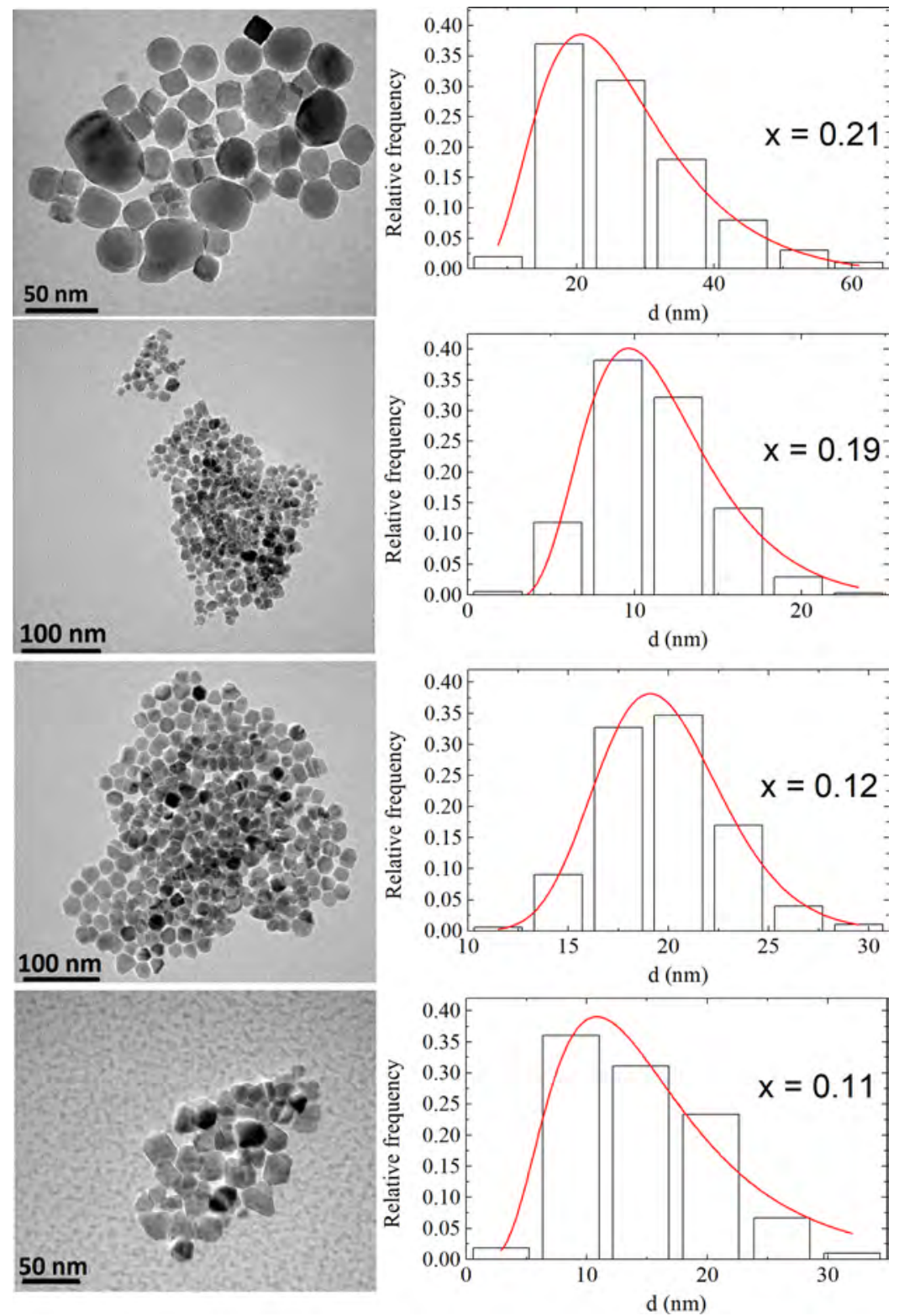

Figure 1. Representative TEM images of the as-prepared $\mathrm{Zn}_{x} \mathrm{Fe}_{3-x} \mathrm{O}_{4} \mathrm{NPs}$. The right panel shows the respective diameter histograms fitted with a lognormal distribution, whose parameters are given in Table 1.

protocols $\left(M_{\mathrm{ZFC}}\right.$ and $M_{\mathrm{FC}}$ curves, respectively) in the SQUID magnetometer with an applied field of $4 \mathrm{kA} / \mathrm{m}$. The blocking temperature distribution $f\left(T_{\mathrm{B}}\right)$ is obtained by the expression $(1 / T) \mathrm{d}\left(M_{\mathrm{ZFC}}(T)-M_{\mathrm{FC}}(T)\right) / \mathrm{d} T$ (see pg. S4 and pg. S5 of Supporting Information), which resulted in good agreement with the blocking temperature distribution obtained from the temperature decay of remanence curve, as shown in a previous work. $^{27}$ Other approximations are used in the literature to obtain the blocking temperature, ${ }^{28-32}$ and the difference between the $f\left(T_{\mathrm{B}}\right)$ obtained from these expressions depends on the dispersion of size distribution; for comparison, one of them $\left[f^{+}\left(T_{\mathrm{B}}\right) \alpha \mathrm{d}\left(M_{\mathrm{ZFC}}(T)-M_{\mathrm{FC}}(T)\right) / \mathrm{d} T\right]$ is compared with the expression $(1 / T) \mathrm{d}\left(M_{\mathrm{ZFC}}(T)-M_{\mathrm{FC}}(T)\right) / \mathrm{d} T$ used in this work (see Figure S4 in pg. S6 of Supporting Information). The samples for magnetic measurements were conditioned by dispersing the NPs in epoxy resin in low concentrations (about $0.1 \mathrm{wt} \%)$, avoiding the agglomeration and physical rotation of the NPs with the applied field.
In order to avoid the thermal dependence of specific heat $\left[c_{p}(T)\right]$ of paraffin, $\mathrm{CBO}$ and $\mathrm{CBO}$-containing NPs at concentrations of $0.2 \mathrm{wt} \%(\mathrm{CBO}+\mathrm{NPs})$ were determined by differential scanning calorimetry (DSC) using a TA Instruments Q2000 calorimeter. A three-step procedure ${ }^{33}$ has been performed at 10,5 , and $1 \mathrm{~K} / \mathrm{min}$, under $50 \mathrm{~mL} / \mathrm{min} \mathrm{N}_{2}$ flow in hermetic aluminum crucibles, using sapphire as a calibration substance. DSC results were analyzed in a similar way than used in ref 23 for $\mathrm{CBO}$ and in ref 16 for paraffin, reflecting the melting process. For $\mathrm{CBO}$, two peaks were observed, one associated with the unresolved LMF and MMF melting and another peak related to the HMF melting. The last transition is the only one developed within the temperature range of hyperthermia experiments $(292-330 \mathrm{~K})$. The enthalpy related to the last peak $\left(\Delta H_{\mathrm{M}}\right)$ was determined as the area of the measured $c_{p}(T)$ curve with respect to the baseline curve $\left[c_{p}^{\text {baseline }}(T)\right]$ as 


$$
\Delta H_{\mathrm{M}}[\mathrm{J} / \mathrm{g}]=\int_{T_{\mathrm{i}}}^{T_{\mathrm{F}}} c_{p}(T) \mathrm{d} T-\int_{T_{\mathrm{i}}}^{T_{\mathrm{F}}} c_{p}^{\text {baseline }} \mathrm{d} T
$$

where $T_{\mathrm{i}}$ and $T_{\mathrm{F}}$ are the initial and final temperatures of the $\mathrm{HMF}$, respectively, defined by the linear extrapolation of the increase and decrease in the initial and final steps of the HMF, respectively. For paraffin, without NPs, the melting process is related to a peak between $307.2 \mathrm{~K}\left(T_{\mathrm{i}}\right)$ and $327.4 \mathrm{~K}\left(T_{\mathrm{F}}\right)$.

The samples used in $\mathrm{MFH}$ experiments were prepared by heating $\mathrm{CBO}$ and paraffin until 317 and $343 \mathrm{~K}$, respectively, with a small amount of toluene solution containing the amount of NPs necessary to obtain the final concentration being reduced. This mixture was exposed to ultrasound for $30 \mathrm{~min}$ at $317 \mathrm{~K}$ for $\mathrm{CBO}$ and at $343 \mathrm{~K}$ for paraffin, both in an open flask. In order to determine the absence of magnetic impurities in the $\mathrm{CBO}$ and paraffin used in the experiments, $M(H)$ curves at $300 \mathrm{~K}$ of both materials without NPs were measured using a VSM (see Figure S5 in pg. S7 of Supporting Information). In both cases, a diamagnetic component was observed, indicating the absence of magnetic contamination, within the detection limits of the experimental technique.

MFH experiments were performed in two different commercial models ( $\mathrm{nB}$ Nanoscale Biomagnetics, Spain), operating with a magnetic field amplitude $\left(H_{0}\right)$ of $16 \mathrm{kA} / \mathrm{m}$ : a DM100-model with a working frequencies $(f)$ of 570 and $817 \mathrm{kHz}$, and a F1-D5 RF-model with $f=570 \mathrm{kHz}$. These apparatuses present different thermal insulation setups and magnetic field gradients produced by the solenoid, the DM100-model having better insulation and a less field gradient. As calibration, $\mathrm{MFH}$ experiments with $\mathrm{CBO}$ and paraffin without NPs were performed at room temperature, showing the absence of temperature increment in both cases (see Figure S6 in pg. S8 of Supporting Information). The quantities $c_{p}(\mathrm{~d} T / \mathrm{d} t)[\mathrm{W} / \mathrm{g}]$ and its integral in the corresponding time interval were numerically calculated using an interpolation in the desired range for both curves after an advanced-averaging smoothing procedure for the measured $T(t)$ curves, being labeled as $\Gamma(t)$

$$
\Gamma(t)[\mathrm{J} / \mathrm{g}]=\int_{t_{0}}^{t} c_{p}\left(\frac{\mathrm{d} T}{\mathrm{~d} t}\right) \mathrm{d} t
$$

\section{RESULTS AND DISCUSSION}

Representative TEM images of synthesized NPs of the four samples are shown in Figure 1, with the respective histograms of diameters presented in the right panel and fitted with a lognormal distribution for all samples. The values of $\langle d\rangle$ and $\sigma$ obtained from the fitting are given in Table 1 .

Concerning the magnetic properties of our samples, they present a distinct mean blocking temperature $T_{\mathrm{B}}$ (see Table 1) obtained from the $M_{\mathrm{ZFC}}(T)$ and $M_{\mathrm{FC}}(T)$ measurements presented in Figure 2. This is a consequence of the expected changes in the effective magnetic anisotropy $K_{\text {eff }}$ and volume of samples. Figure 2 also shows the corresponding blocking temperature distribution $f\left(T_{\mathrm{B}}\right)$ of all samples, according to the established results presented in pgs. S4 and S5 of Supporting Information, compared with the blocking temperature distribution $f^{*}\left(T_{\mathrm{B}}\right)$ calculated from the diameter histogram obtained from TEM data (Figure 1) by Néel's model assuming a value of $\tau_{0}=10^{-10} \mathrm{~s}$, measuring time $\tau_{\mathrm{m}}=100 \mathrm{~s}$ and using the amplitude and energy barrier as fitting parameters. $K_{\text {eff }}$ was calculated through the definition of blocking temperature in Néel's model by matching the distribution from TEM results
Table 1. NP Size Distribution and Magnetic Parameters of $\mathrm{Zn}_{x} \mathrm{Fe}_{3-x} \mathrm{O}_{4}$ Obtained from TEM Images and Magnetization Measurements

$\begin{array}{cccccc}X & { }^{a}\langle d\rangle(\mathrm{nm}) & { }^{a} \sigma & { }^{b} M_{\mathrm{S}}^{300 \mathrm{~K}}(\mathrm{kA} / \mathrm{m}) & { }^{c}\left\langle T_{\mathrm{B}}\right\rangle(\mathrm{K}) & { }^{d} K_{\text {eff }}\left(\mathrm{J} / \mathrm{m}^{3}\right) \\ 0.21 & 24.7 & 0.41 & 74 & 55 & 3.0 \times 10^{3} \\ 0.19 & 11.0 & 0.18 & 75 & 32 & 2.5 \times 10^{3} \\ 0.12 & 19.7 & 0.36 & 69 & 72 & 5.2 \times 10^{3} \\ 0.11 & 14.0 & 0.50 & 63 & 64 & 7.5 \times 10^{3}\end{array}$

${ }^{a}\langle d\rangle$ is the mean particle size and $\sigma$ is the size dispersion obtained from the fitting of the diameter histogram with a lognormal. ${ }^{b} M_{\mathrm{S}}^{300 \mathrm{~K}}$ is the saturation magnetization at $300 \mathrm{~K}$ obtained from the hysteresis cycle. ${ }^{c}\left\langle T_{B}\right\rangle$ is the mean blocking temperature obtained from the blocking temperature distribution $f\left(T_{\mathrm{B}}\right)$ calculated from the $M_{\mathrm{ZFC}}$ and $M_{\mathrm{FC}}$ curves. ${ }^{d} K_{\text {eff }}$ is the effective magnetic anisotropy obtained from the fit of $f\left(T_{\mathrm{B}}\right)$ with the blocking temperature distribution calculated from the diameter histogram $f^{*}\left(T_{\mathrm{B}}\right)$.

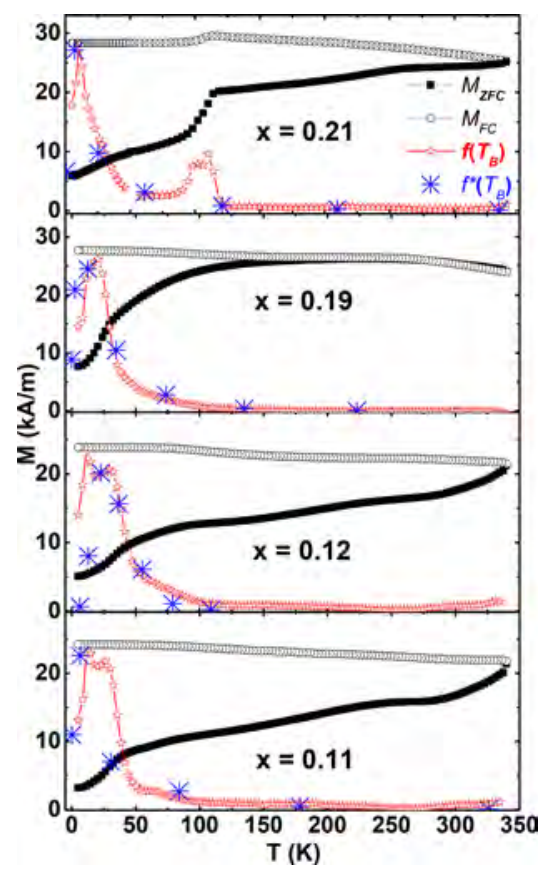

Figure 2. $M_{\mathrm{ZFC}}(T)$ and $M_{\mathrm{FC}}(T)$ curves of $\mathrm{Zn}_{x} \mathrm{Fe}_{3-x} \mathrm{O}_{4} \mathrm{NPs}$ (solid and open squares, respectively) measured with an applied field of $4 \mathrm{kA} / \mathrm{m}$. The respective blocking temperature distribution $f\left(T_{\mathrm{B}}\right)$ (red stars) is also presented together with $f^{*}\left(T_{\mathrm{B}}\right)$ (blue crosses), as calculated from the size distributions given in Figure 1.

and the $f\left(T_{\mathrm{B}}\right)$ distribution from magnetic measurements. The obtained $K_{\text {eff }}$ values are presented in Table 1. A high irreversibility temperature is observed in all curves with the exception of sample $x=0.19$. These high irreversibility temperatures indicate the contribution of NPs with a large anisotropy but not so relevant in population.

Table 1 also gives the saturation magnetization values $\left(M_{\mathrm{S}}\right)$ measured at $300 \mathrm{~K}$ for all samples. Magnetization curves as a function of applied field $M(H)$ are shown in Figure S3, pg. S3 of Supporting Information. Notice that sample $x=0.19$ presents a composition and morphology resulting in a product $K_{\text {eff }} d^{3}$ with relatively narrow size dispersion close to a value that results in reasonable SPA values. For the other samples, the product $K_{\text {eff }} d^{3}$ would result in a reduced SPA value and, in this way, the broader size distributions of these samples result in a SPA that allows performing our $\mathrm{MFH}$ experiments in the temperature range of phase transition in both media. 

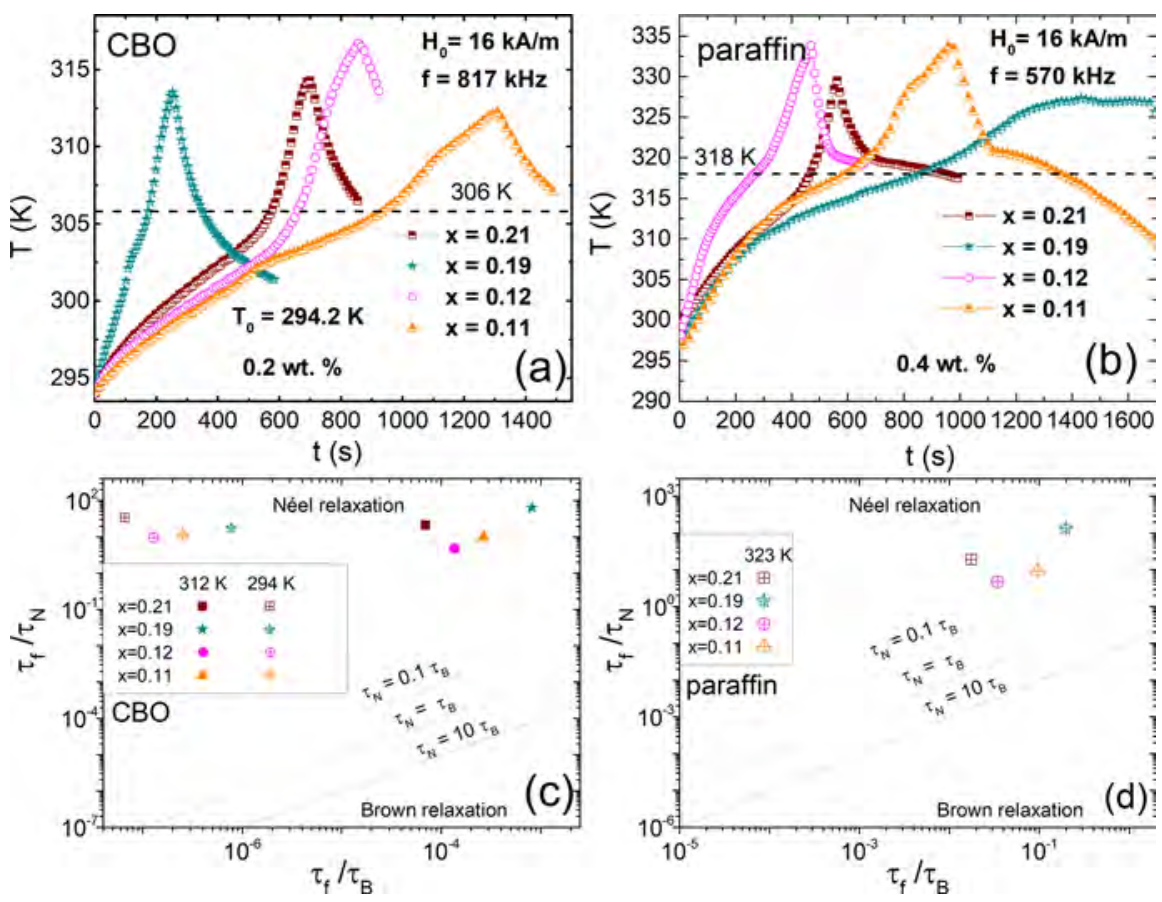

Figure 3. (a,b) Evolution of temperature with time during the MFH experiments performed in the DM100-model for $0.2 \mathrm{wt} \%$ of samples $x=0.21$, $0.19,0.12$, and 0.11 dispersed in $\mathrm{CBO}\left(H_{0}=16 \mathrm{kA} / \mathrm{m}\right.$ and $\left.f=817 \mathrm{kHz}\right)$ and in paraffin $\left(H_{0}=16 \mathrm{kA} / \mathrm{m}\right.$ and $\left.f=570 \mathrm{kHz}\right)$, respectively. Dotted line indicates the temperatures 306 and $318 \mathrm{~K}$, where an increase in the slope is observed for $\mathrm{CBO}$ and paraffin, respectively. (c,d) Relaxation time diagram, where the Néel $\left(\tau_{\mathrm{N}}\right)$ and Brown $\left(\tau_{\mathrm{B}}\right)$ relaxation times of different NPs dispersed in CBO and in paraffin, respectively, normalized by the applied field period $\left(\tau_{\mathrm{f}}=1 /(2 \pi f)\right)$ are plotted; latter data were calculated for two temperatures 294 and $312 \mathrm{~K}$, where the CBO presents different viscosities, and $323 \mathrm{~K}$, above the melting point of paraffin.

Figure 3a shows the results of the MFH experiment of all samples of NPs dispersed in $\mathrm{CBO}$ at a concentration of $0.2 \mathrm{wt}$ $\%$ performed with $H_{0}=16 \mathrm{kA} / \mathrm{m}$ and $f=817 \mathrm{kHz}$ from the initial temperature $T_{0}=294.2 \mathrm{~K}$ in the DM100-model. As expected, the samples present a distinct heating rate, which is a consequence of different relaxation times resulting from different morphologies, specifically $\langle d\rangle$ and $\sigma$, and compositions, which is reflected in different $\mathrm{M}_{\mathrm{S}}$ and anisotropy energy barriers proportional to $\left\langle T_{\mathrm{B}}\right\rangle$. Figure $3 \mathrm{~b}$ gives the MFH results of the DM100-model $\left(H_{0}=16 \mathrm{kA} / \mathrm{m}\right.$ and $\left.f=570 \mathrm{kHz}\right)$ for the samples $x=0.21,0.19,0.12$, and 0.11 dispersed in paraffin at a concentration of 0.4 wt $\%$, which present similar trends to the samples dispersed in $\mathrm{CBO}$, except that the increment in the heating rate is observed at a higher temperature $(\sim 318 \mathrm{~K})$.

Usually, SPA is obtained from the initial slope of these curves by the simplified relationship SPA $=\left(m / m_{\mathrm{NPs}_{\mathrm{s}}}\right) c_{p}(\mathrm{dT} /$ $\mathrm{d} t)$, where $c_{p}$ and $m$ are the specific heat and the mass of the media, respectively, and $m_{\mathrm{NPs}}$ is the mass of the NPs. This expression assumes that all energy absorbed by the NPs from the ac applied field is used to increase the temperature, and heat losses are negligible during the first few seconds of heating. After the initial seconds, however, the heating rate $(\mathrm{d} T / \mathrm{d} t)$ is observed to depart from the linear behavior due to nonadiabaticity of the system. However, in these systems of NPs dispersed in $\mathrm{CBO}$ and in paraffin, the heating curves reveal a complex behavior with regions with different heating rates, which increases around $306 \mathrm{~K}$ for all samples in $\mathrm{CBO}$ and around $318 \mathrm{~K}$ for paraffin, as indicated by the dotted lines in Figure 3a,b.

The increase in the heating rate during the MFH experiment observed in Figure $3 a, b$ could be due to changes in the thermodynamic properties of media or a change in the NP relaxation mechanism, from Néel to Brown driven by the melting of $\mathrm{CBO}$ (or paraffin), and the consequent reduction in the viscosity $\eta$ of CBO (in the case of paraffin, a change from the solid to liquid state). In order to evaluate the possibility of change in the relaxation mechanism as a consequence of $\mathrm{CBO}$ melting, we calculate the Brown $\left(\tau_{\mathrm{B}}\right)$ and Néel $\left(\tau_{\mathrm{N}}\right)$ relaxation times of all samples. The characteristic times were calculated as $\tau_{\mathrm{B}}=3 \eta V_{\text {hyd }} / k_{\mathrm{B}} T$ and $\tau_{\mathrm{N}}=\tau_{0} \exp \left(27\left\langle T_{\mathrm{B}}\right\rangle / T\right)$, using $\tau_{0}=10^{-10}$ $\mathrm{s}$, the volume obtained from TEM, the CBO viscosity $\eta=10$ and $10 \mathrm{mPa} \cdot \mathrm{s}$ for $T=294$ and $312 \mathrm{~K}$, the lower and higher temperatures than the $\mathrm{CBO}$ melting point, respectively. We consider $V_{\text {hyd }}=(\pi / 6)\langle d\rangle^{3}$, without including an organic layer usually present in the NPs prepared by this method and, therefore, the value of $\tau_{\mathrm{B}}$ is underestimated. The relationship between $\tau_{\mathrm{N}}$ and $\tau_{\mathrm{B}}$ is plotted in the relaxation time diagrams built according to Lima et al. ${ }^{2}$ in Figure $3 \mathrm{c}$. From this figure, it is clear that $\tau_{\mathrm{N}}<\tau_{\mathrm{B}}$ (even considering an underestimated value of $\tau_{\mathrm{B}}$ ) above and below the melting temperature. In this way, the magnetic relaxation mechanism is the dominant one for all samples and in all the studied temperature range. This result rules out the hypothesis that a change in the NP relaxation mechanism could be responsible for the increase in the heating rate in the $\mathrm{MFH}$ experiments during the $\mathrm{CBO}$ melting. Figure $3 \mathrm{~d}$ presents a similar diagram for the NPs in paraffin $(\eta=2.1$ $\mathrm{mPa} \cdot \mathrm{s}$ ) for $T=353 \mathrm{~K}$, resulting in $\tau_{\mathrm{N}}<\tau_{\mathrm{B}}$ for the four samples and reflecting the Neel relaxation mechanisms as the dominant one.

Figure $4 \mathrm{a}$ presents the $c_{p}(T)$ curves of CBO measured at different heating rates: 1 and $10 \mathrm{~K} / \mathrm{min}$. As is clearly observed, there are two peaks in both curves, which are better defined for the highest heating rate. The first peak is associated with the unresolved LMF and MMF transitions at lower temperatures, 

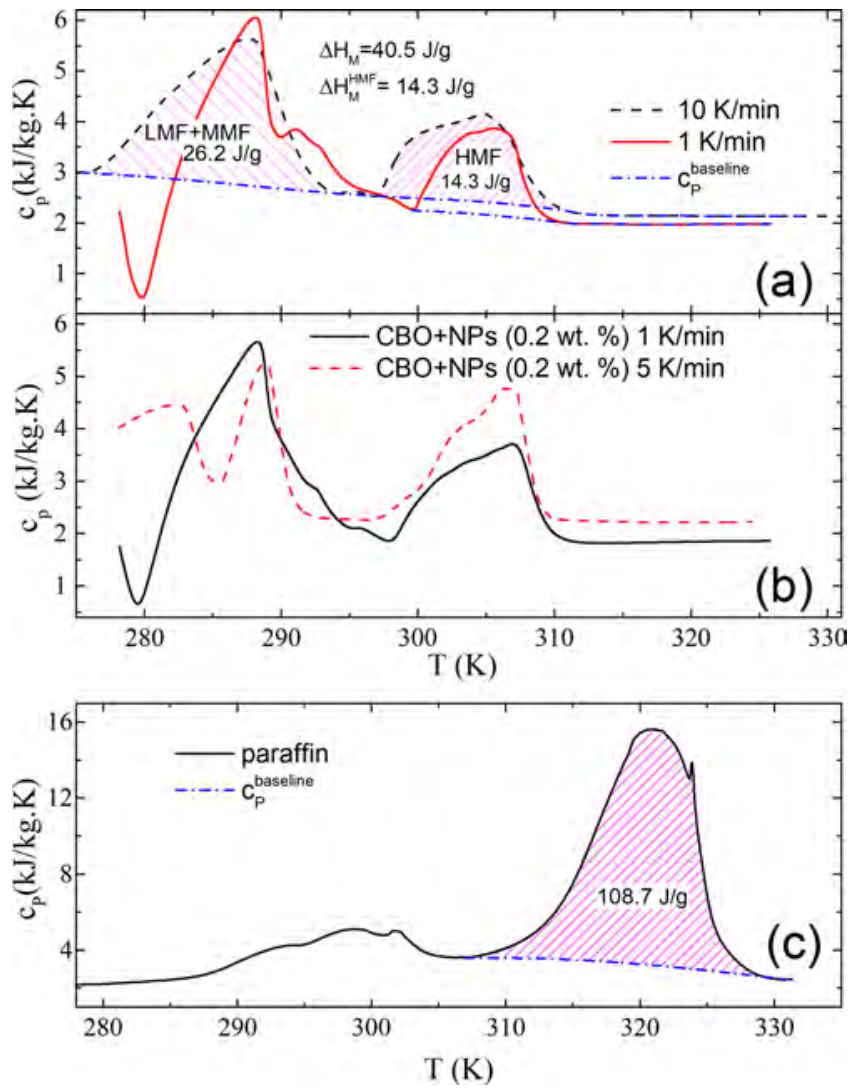

Figure 4. (a) Thermal dependence of specific heat $\left[c_{p}(T)\right]$ of $\mathrm{CBO}$ measured with heating rates of 1 and $10 \mathrm{~K} / \mathrm{min}$, as well as of the $c_{p}^{\text {baseline }}(T)$ curves (dotted-blue line for both heating rates). Dashed zones indicate the difference between $c_{p}(T)$ and $c_{p}^{\text {baseline }}(T)$ associated with the melting of distinct fractions. (b) $c_{p}(T)$ curves for $\mathrm{CBO}$ containing $0.2 \mathrm{wt} \%$ of $x=0.19 \mathrm{NPs}$ with heating rates of 1 and $5 \mathrm{~K} /$ min. (c) $c_{p}(T)$ curves for paraffin with a heating rate of $10 \mathrm{~K} / \mathrm{min}$.

and the second peak is associated with the HMF transition of $\mathrm{CBO}^{23}$ The effect of total melting transition can be observed by the naked eye, as shown in the pictures taken at different temperatures (see Figure S7 in pg. S9 of Supporting Information), where the melting is complete after the HMF transition. As mentioned before, the MFH experiments are performed in the temperature range where the HMF transition develops. The temperature range of the HMF peak $\left(\Delta T_{M}\right)$ is well marked for both heating rates and can be calculated from the extrapolated peak onset and offset temperatures in the $c_{p}(T)$ curves: $297.1-310.8 \mathrm{~K}$ for $10 \mathrm{~K} / \mathrm{min}$ and $299.8-309.0 \mathrm{~K}$ for $1 \mathrm{~K} / \mathrm{min}$. For the heating rate of $10 \mathrm{~K} / \mathrm{min}$, where the peaks are better defined, a melting enthalpy value of $40.5 \mathrm{~J} / \mathrm{g}$ is calculated using eq 1 and taking the area of both peaks, that is, for the whole melting process (LMF + MMF and HMF).

Figure $4 \mathrm{~b}$ gives the $c_{p}(T)$ curves measured with heating rates of 1 and $5 \mathrm{~K} / \mathrm{min}$ for $\mathrm{CBO}$ containing $x=0.19 \mathrm{NPs}$ at the concentration of $0.2 \mathrm{wt} \%$. As observed, these curves present differences among them and with respect to the curves of pure CBO. Notice that the presence of NPs results in smaller changes in $\Delta T_{\mathrm{M}}$ than those caused by different heating rates in pure $\mathrm{CBO}$. For the heating rates of 1 and $5 \mathrm{~K} / \mathrm{min}, \Delta T_{\mathrm{M}}$ of the $\mathrm{HMF}$ is between 297 and $310 \mathrm{~K}$. For the analysis of $\mathrm{MFH}$ experiment, the $c_{p}(T)$ curves reported in Figure 4a,b were used for pure $\mathrm{CBO}$ and for $\mathrm{CBO}$-containing NPs, respectively.
Finally, Figure $4 c$ gives the $c_{p}(T)$ curve for the paraffin measured with a heating rate of $10 \mathrm{~K} / \mathrm{min}$.

Independent from the effects of NPs and heating rate, the important changes in the $c_{p}(T)$ curve of CBO should be taken into account for the analysis of the MFH results. In particular, the estimation of the SPA of the system should be revised when the specific heat presents a nonmonotonous evolution, and latent heat is stored or released during the phase transition. In this way, the thermal energy of the system when the applied field is turned on and off can be written, respectively, as

$$
\begin{aligned}
& m_{\mathrm{CBO}} c_{p}(T) \mathrm{d} T=\mathrm{d} Q_{\mathrm{SPA}}(T)-\mathrm{d} Q_{\text {loss }}(T) \quad\left(H_{0}>0\right) \\
& m_{\mathrm{CBO}} c_{p}(T) \mathrm{d} T=-\mathrm{d}_{\text {loss }}(T) \quad\left(H_{0}=0\right)
\end{aligned}
$$

where $m_{\mathrm{CBO}}$ is the mass of the medium (CBO), $Q_{\mathrm{SPA}}$ is the heat released by the magnetic losses of NPs for $H_{0}>0$, and $Q_{\text {loss }}(T)$ are the heat losses of system due to the nonadiabatic condition of the MFH experimental apparatus. ${ }^{34}$ The amount of $Q_{\text {loss }}$ depends on the MFH apparatus used. We remark again that, according to our measurements of cooling down curves (after the applied field is turned off), $Q_{\text {loss }}$ is lower for the DM100-model than for the F1-D5-model. In addition, $Q_{\text {loss }}(T)$ is expected to depend on the temperature difference between the sample and the environment. The melting enthalpy is considered in the thermal variation of $c_{p}(T)$ through eq 1 .

Taking into account the appropriate $c_{p}(T)$ curve together with eqs 3 and 4, the hyperthermia curves could be divided into four regions: A, B, and $\mathrm{C}$, where the applied field is on $\left(\mathrm{H}_{0}\right.$ $>0$ ) and $D$, where $H_{0}=0$. Figure 5a shows these four regions for sample $x=0.21$ (at a concentration of 0.2 wt $\%, f=817$ $\mathrm{kHz}$, and $\left.H_{0}=16 \mathrm{kA} / \mathrm{m}\right)$ using the DM100-model. Region $\mathrm{A}$ is in the temperature range below the HMF transition $(T<297$ $\mathrm{K}), \mathrm{B}$ coincides with the HFM melting $(297 \mathrm{~K}<T<309 \mathrm{~K})$, and $\mathrm{C}$ is for temperatures above the HMF region $(T>309 \mathrm{~K})$. As discussed previously, the increase in the MFH curve above $306 \mathrm{~K}$ could not be associated with a change in the dominant relaxation mechanism due to the strong reduction in the viscosity of $\mathrm{CBO}$ above the melting point. In fact, a change in the $\mathrm{CBO}$ physical state is observed in the pictures at $307 \mathrm{~K}$ compared with that at $305 \mathrm{~K}$ (see Figure S7 in pg. S9 of Supporting Information). Also, the reduction in the viscosity of CBO, some degrees below $T_{\mathrm{F}}=309 \mathrm{~K}$, may induce some changes in the spatial arrangement of NPs, as discussed below.

Integration of eqs 3 and 4 in the corresponding interval of temperature results in

$$
\begin{aligned}
& \int_{T_{0}}^{T} c_{p}(T) \mathrm{d} T=\int_{t_{0}}^{t} c_{p}(T)\left(\frac{\mathrm{d} T}{\mathrm{~d} t}\right) \mathrm{d} t=\frac{\left(Q_{\mathrm{SPA}}-\Delta Q_{\mathrm{loss}}\right)}{m_{\mathrm{CBO}}} \\
& \left(H_{0}>0\right) \\
& \int_{T_{0}}^{T} c_{p}(T) \mathrm{d} T=\int_{t_{0}}^{t} c_{p}(T)\left(\frac{\mathrm{d} T}{\mathrm{~d} t}\right) \mathrm{d} t=\frac{-\Delta Q_{\text {loss }}}{m_{\mathrm{CBO}}} \\
& \left(H_{0}=0\right)
\end{aligned}
$$

For eq 5 , the integral limits correspond to the starting time $t_{0}$ and the final $t$, where the field is turned off, with the corresponding temperatures $T_{0}$ and $T$, respectively. For eq 6 , these integral intervals represent the time and temperature ranges of region $D$, where $H_{0}=0$. Thus, eq 5 gives information 


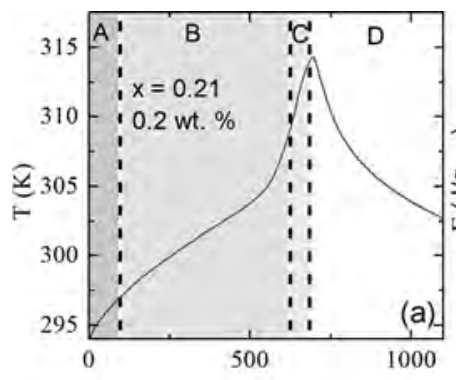

$\mathrm{t}(\mathrm{s})$

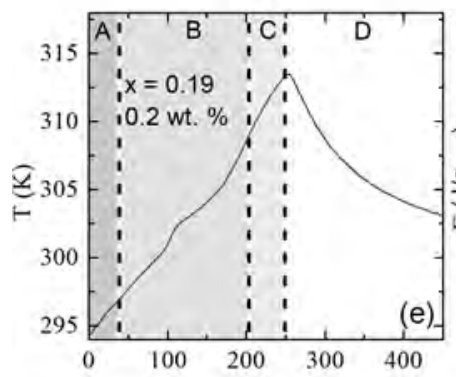

$\mathrm{t}(\mathrm{s})$

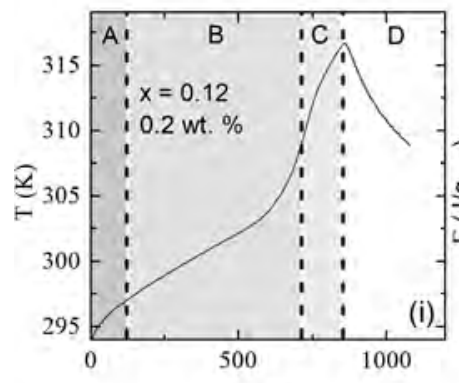

$\mathrm{t}(\mathrm{s})$

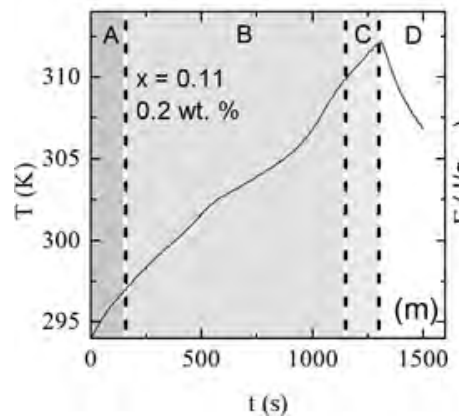

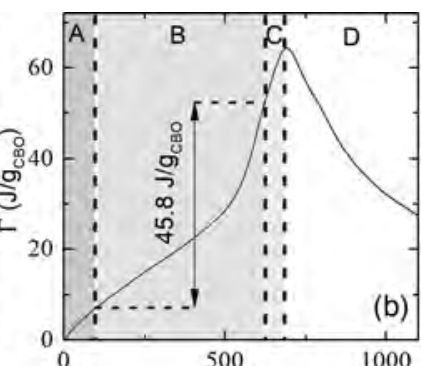

$\mathrm{t}(\mathrm{s})$

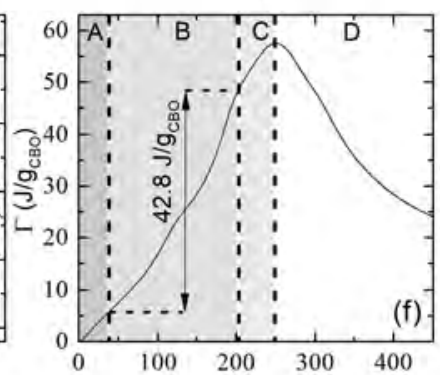

$\mathrm{t}(\mathrm{s})$

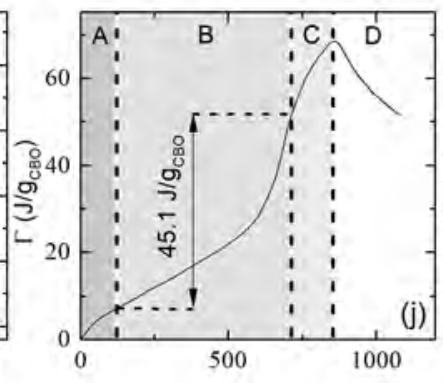

$t(s)$

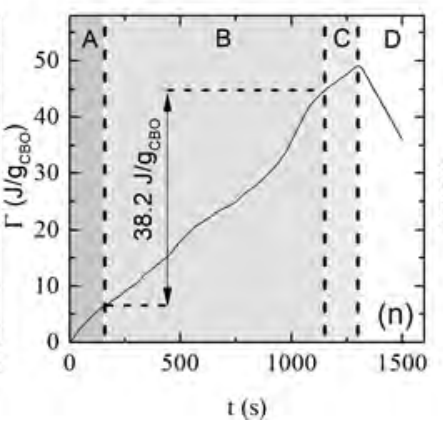

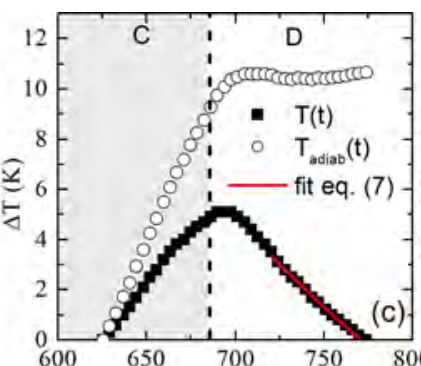

$\mathrm{t}(\mathrm{s})$

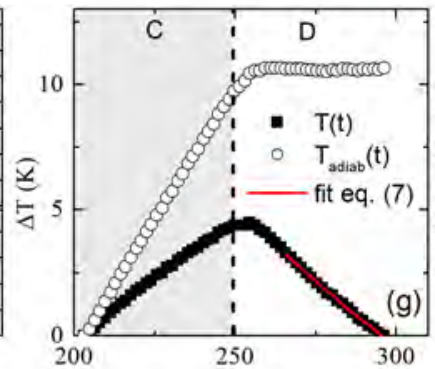

$t(s)$

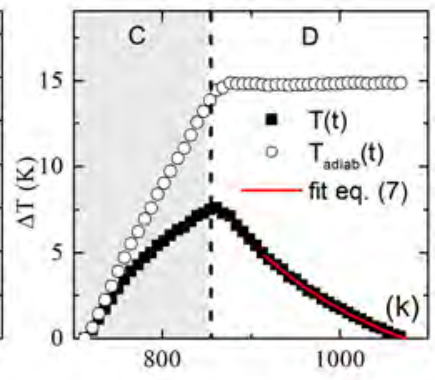

$t(s)$

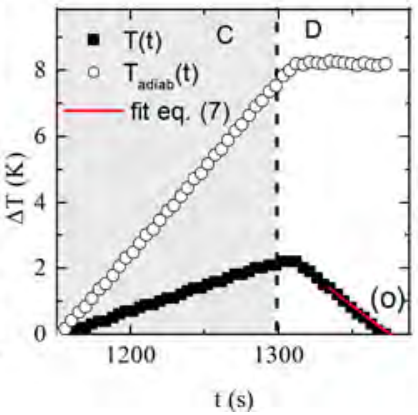

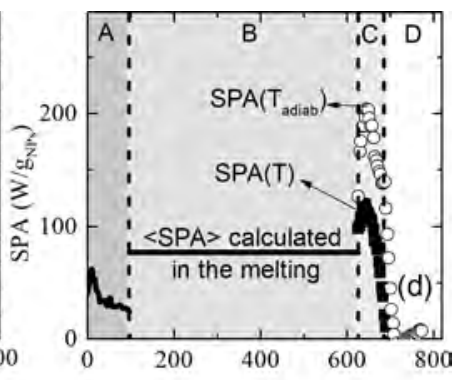

$\mathrm{t}(\mathrm{s})$

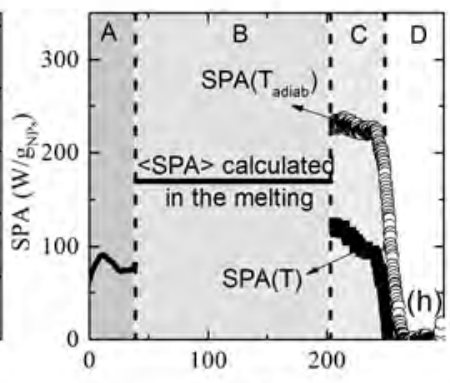

$t(s)$

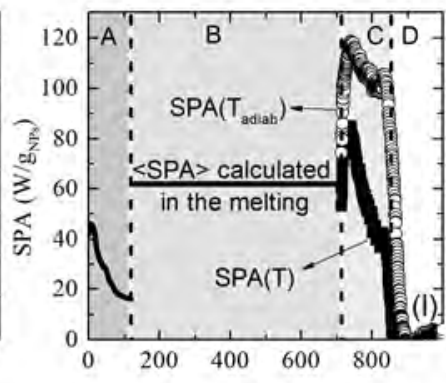

$\mathrm{t}(\mathrm{s})$

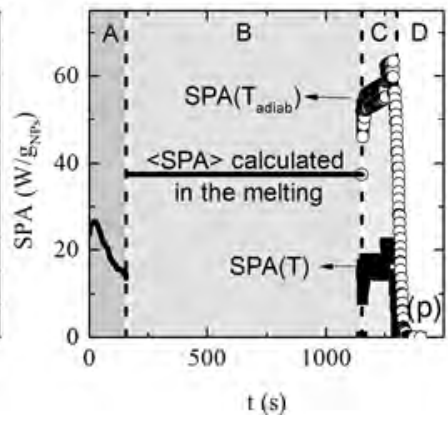

Figure 5. (a) $T(t)$ results of MFH experiments for CBO containing $0.2 \mathrm{wt} \%$ of $x=0.21 \mathrm{NPs}$ with $f=817 \mathrm{kHz}$ and $H_{0}=16 \mathrm{kA} / \mathrm{m}$ in the DM100model. Regions A, B, and C correspond to the temperature range defined below, within, and above the HMF peak in the $c_{p}(T)$ curve associated with the melting of $\mathrm{CBO}+$ NPs. (b) $\Gamma(t)$ curves obtained from the $\mathrm{MFH}$ results and the line gives the heat variation within the intervals $T_{\mathrm{i}}$ and $T_{\mathrm{F}}$. (c) $\Delta T(t)=T(t)-T_{0}$ curve measured for region C fitted with eq 7 and the temperature corrected for adiabatic conditions $\left[\Delta T_{\text {adiab }}(t)=T_{\text {Adiab }}(t)\right.$ $-T_{0}$ ] calculated using eq 8. (d) Estimated SPA values for regions A and C, as well as the mean SPA (〈SPA $\rangle$ ) estimated for region B. Similar curves for samples $x=0.19, x=0.12$, and $x=0.11$ in the same concentration are presented in $(\mathrm{e}-\mathrm{p})$, respectively.

about the SPA in regions $\mathrm{A}, \mathrm{B}$, and $\mathrm{C}$, while eq 6 provides information on $\Delta Q_{\text {loss }}$.

Figure $5 \mathrm{~b}$ gives the corresponding temporal dependence of $\Gamma(t)$ (eq 2) calculated from the experimental data of $\mathrm{MFH}$ (Figure $5 \mathrm{a}$ ) and $c_{p}(T)$ with a heating rate of $5 \mathrm{~K} / \mathrm{min}$ (see Figure $4 \mathrm{~b}$ ). This graph gives the accumulated thermal energy in $\mathrm{CBO}$, normalized by its mass, due to the magnetic losses from the applied field and the heat loss due to nonadiabatic conditions. This curve is also divided into four regions, taking the corresponding time when the corresponding temperature of Figure 5a is reached. The increase in the heating rate above $306 \mathrm{~K}$ is also observed in these curves.

For region $\mathrm{A}$, it is expected that $\Delta Q_{\text {loss }}$ has a relatively low value in comparison to $Q_{\mathrm{SPA}}$ as heat losses by the nonadiabatic conditions are small at temperatures close to the ambient temperature $\left(T_{\mathrm{e}}=293.7 \mathrm{~K}\right)$. Thus, for region $\mathrm{A}$, the SPA value can be approximated by the quantity $\left(m_{\mathrm{CBO}} / m_{\mathrm{NPs}}\right) c_{\mathrm{p}}(T)(\mathrm{d} T /$ $\mathrm{d} t$ ). In fact, a relatively small decrease of the SPA is observed with the increment of time, probably related with a small value $\Delta Q_{\text {loss }}$ that increases with increasing temperature. 

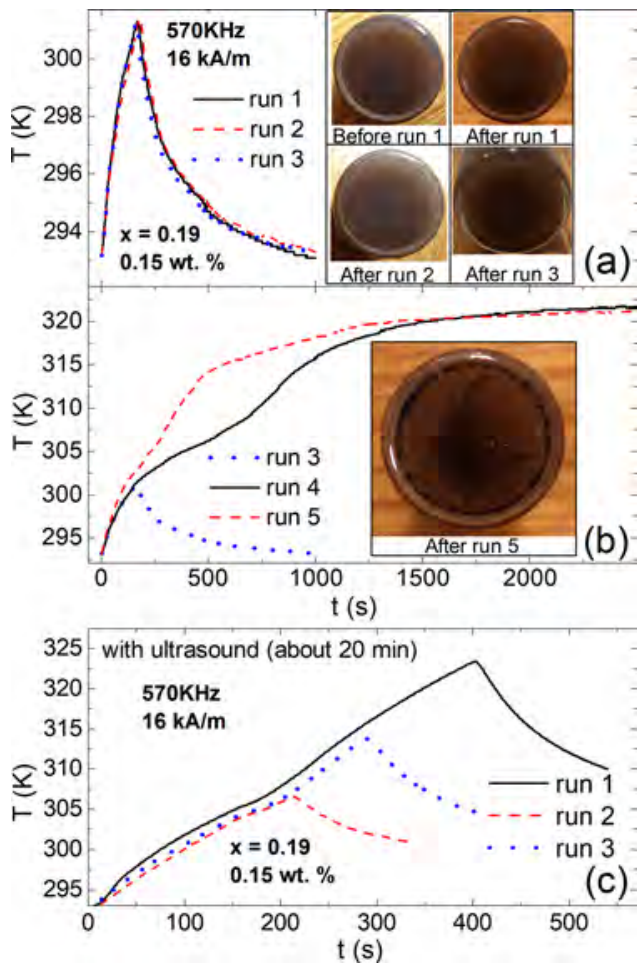

Figure 6. (a) MFH results for three subsequent runs performed in the D5-F1-model $\left(f=570 \mathrm{kHz}\right.$ and $\left.H_{0}=16 \mathrm{kA} / \mathrm{m}\right)$ for CBO containing $0.15 \mathrm{wt} \%$ of $x=0.19 \mathrm{NPs}$ from $T_{0}$ to a final temperature below 306 $\mathrm{K}$, followed by cooling to $T_{0}$. (b) Two subsequent runs (4 and 5) measured under the same experimental conditions and performed from $T_{0}$ to a final temperature above the melting temperature $(T>$ $T_{\mathrm{F}}$ ). Insets present the picture of samples after the indicated run. (c) Three successive runs of the same sample measured in the D5-F1 model $\left(f=570 \mathrm{kHz}\right.$ and $\left.H_{0}=16 \mathrm{kA} / \mathrm{m}\right)$ with intermediate exposure to ultrasound (about $20 \mathrm{~min}$ ).

For region $\mathrm{C}$, this approach cannot be used as the temperature of the sample is considerably higher than $T_{\mathrm{e}}$, and $Q_{\text {loss }}$ should be taken into account. In this way, we propose a correction of the $\mathrm{MFH}$ temperature considering the $Q_{\text {loss }}$ obtained from the cooling curve, when the magnetic field is turned off in order to estimate the SPA in region C. The $T(t)$ data in region $\mathrm{D}$ were fitted with the following expression that reflects their expected exponential decay with time

$$
T(t)=T_{\mathrm{e}}+\left[\left(T_{t_{0}}-T_{\mathrm{e}}\right) \exp \left(-\beta\left(t-t_{0}\right)\right)\right]
$$

where $T_{t_{0}}$ is the value of temperature at time $t_{0}$ (moment when the field is turned off in this region) and $\beta$ is the parameter that models the heating loss. $T_{\mathrm{e}}$ is obtained from the lowtemperature range of the cooling curve of $\mathrm{CBO}$ containing 0.2 wt \% of NPs (see Figure S8 in pg. S10 of Supporting Information). The result of this fitting procedure is given in Figure $5 \mathrm{c}$. From this fitting, the corrected temperature, that is, the temperature expected for adiabatic conditions, $T_{\text {adiab, }}$ is calculated from the measured temperature $T(t)$ as

$$
T_{\text {adiab }}(t)=T(t)+\beta\left[\int_{t_{0}}^{t} T(t) \mathrm{d} T-T_{\mathrm{e}}\left(t-t_{0}\right)\right]
$$

Both $\Delta T_{\text {adiab }}(t)=T_{\text {adiab }}(t)-T_{0}(t)$ and $\Delta T(t)=T(t)-$ $T_{0}(t)$ curves are presented in Figure $5 c$, together with the fitting of the cooling region. As expected, $\Delta T_{\text {adiab }}(t)$ presents an almost constant value in region $\mathrm{D}$, as is expected for a system that does not exhibit losses. This result reinforces the fact that our temperature correction procedure is correct. In this way, the corrected value of SPA can be calculated for region $\mathrm{C}$ using $\left(m_{\mathrm{CBO}} / m_{\mathrm{NPS}}\right) c_{\mathrm{p}}(T)\left(\mathrm{d} \Delta T_{\text {adiab }}(t) / \mathrm{d} t\right)$.

For region $\mathrm{B}$, fitting the SPA dependence with time is not simple because of the HMF melting within this temperature range. Nevertheless, it is possible to estimate a mean SPA value $(\langle\mathrm{SPA}\rangle)$ using eq 5 as follows: (i) the left side of eq 5 in the time interval $T_{\mathrm{i}} \leq T \leq T_{\mathrm{F}}$ (see eq 1 ) could be obtained directly from the data presented in Figure $5 \mathrm{~b}$, as indicated, being 45.8 $\mathrm{J} / \mathrm{g}_{\text {Сво }}$ (ii) the quantity $\Delta Q_{\text {loss }}$ can be estimated from the equivalent curve from the cooling of $\mathrm{CBO}+\mathrm{NPs}$ until the temperature attains $T_{\mathrm{e}}$ (see Figure $\mathrm{S} 8$ in pg. $\mathrm{S} 10$ of Supporting Information), being $36.3 \mathrm{~J} / \mathrm{g}_{\mathrm{CBO}}$. Therefore, taking these values into account, the mean value of $\langle\mathrm{SPA}\rangle$ is found to be $77 \mathrm{~W} /$ $\mathrm{g}_{\mathrm{NPs}}$. This $\langle\mathrm{SPA}\rangle$ value is shown in Figure $5 \mathrm{~d}$, together with the values of SPA obtained for regions $A$ and $C$ (here the values obtained for $\Delta T(\mathrm{t})$ using the same expression are also included, i.e., uncorrected by $Q_{\text {loss }}$ ). Similar analysis was performed for samples $x=0.19$ (Figure $5 \mathrm{e}-\mathrm{h}$ ), $x=0.12$ (Figure $5 \mathrm{i}-1$ ), and $x=0.11$ (Figure $5 \mathrm{~m}-\mathrm{p}$ ). Sample $x=0.19$ presents a higher heating rate in MFH experiments when compared with sample $x=0.21$, resulting in the following less evident changes: the decrease of SPA in region A with increasing temperature and a less pronounced decrease in the heating rate between $T_{\mathrm{i}}=297 \mathrm{~K}$. In contrast, samples $x=0.12$ and $x=0.11$ have a smaller heating rate in comparison with sample $x=0.21$, making the changes mentioned above more pronounced.

The $\langle$ SPA $\rangle$ values estimated for region $\mathrm{B}$ has an intermediate value between those obtained for regions $A$ and $C$ in all samples. Probably, the SPA is not constant along this region, with a probable increment above $306 \mathrm{~K}$. The decrease in the heating rate of $\mathrm{MFH}$ curves between 297 and $306 \mathrm{~K}$ could be probably associated with two contributions. First, the increase of $\Delta Q_{\text {oss }}$ with respect to $Q_{S P A}$ with increasing temperature, which could be observed as the decrease of SPA with increasing temperature in region $\mathrm{A}$, is more evident for samples with lower SPA. Second, the latent heat of $\mathrm{CBO}$ melting reduces the effects of $Q_{S P A}$ with temperature increment. It is more evident for samples with smaller $Q_{S P A}$.

SPA in region $C$ increases between 2.5 and 3 times for all samples with respect to the values in region $A$, corresponding to the clearly noticed increment in the heating rate observed in the $T(t)$ curves measured in MFH experiments. This increment in the SPA for region $\mathrm{C}$ is reflected in the increased slope of the MFH results above $306 \mathrm{~K}$, some degrees below the ending of the melting and corresponding to a physical change in the $\mathrm{CBO}$, with a strong reduction in the viscosity. Despite the reduction of viscosity with melting, $\tau_{\mathrm{N}}$ should be several orders of magnitude smaller than $\tau_{\mathrm{B}}$ (see Figure $3 \mathrm{~b}$ ), which is reinforced by the agglomeration process discussed below. However, the phase transition of $\mathrm{CBO}$ with the consequent reduction in the viscosity allows other changes in the sample with consequences in the magnetic response of the system, as discussed below. The SPA in region $\mathrm{C}$ remains almost constant until the applied field is turned off for samples $x=0.19$ and $x=$ 0.11 . For samples $x=0.21$ and $x=0.12$, the increment in the SPA is slightly slower and a decrease in the SPA value is observed before the applied field is turned off. Interestingly, the SPA in region $\mathrm{C}$ is almost constant for smaller NPs, and it decreases with time for the two larger ones, which could also be related to an agglomeration or precipitation phenomenon 

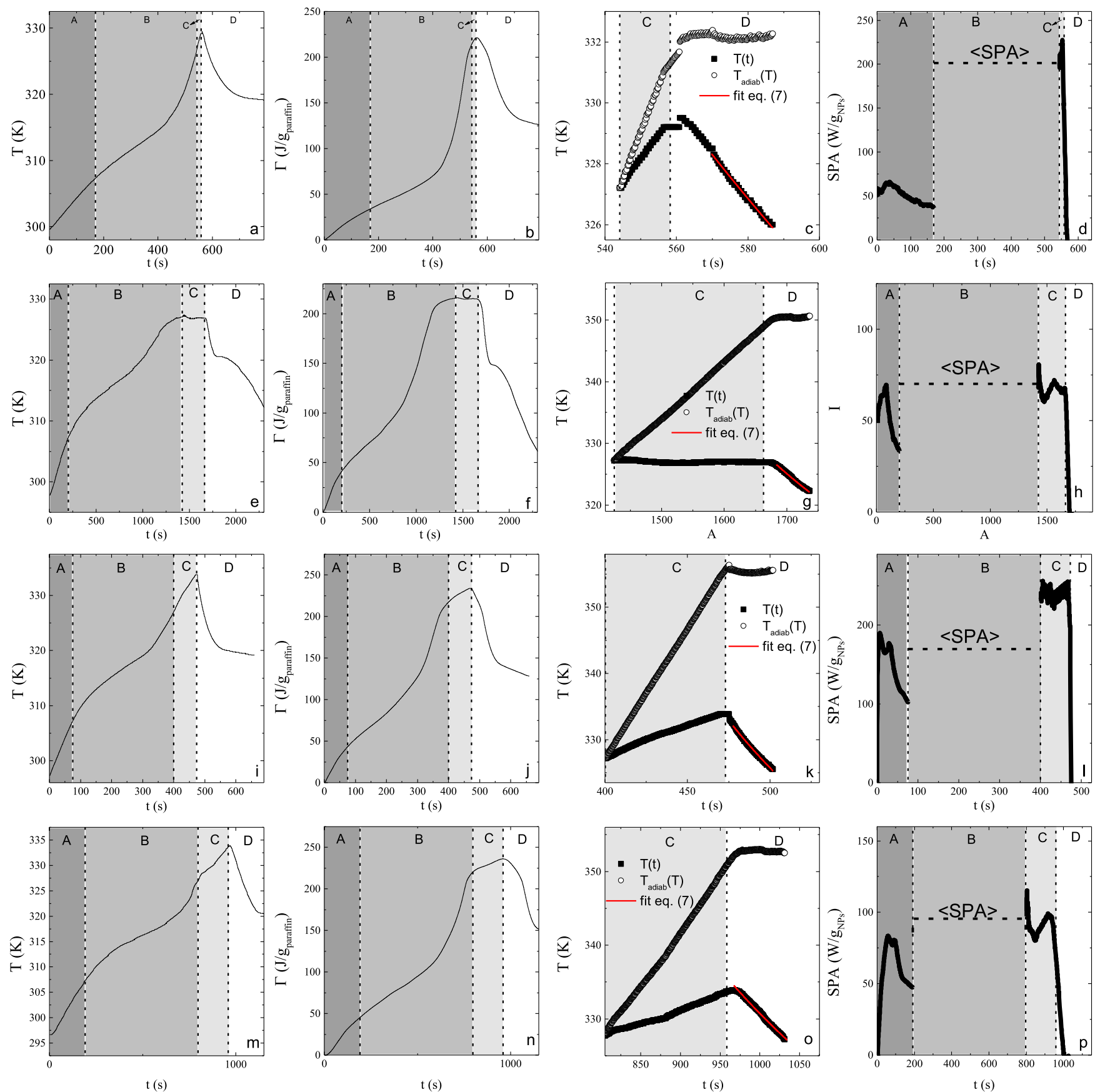

Figure 7. (a) $T(t)$ results of MFH experiments for paraffin containing 0.4 wt $\%$ of $x=0.21 \mathrm{NPs}$ with $f=570 \mathrm{kHz}$ and $H_{0}=16 \mathrm{kA} / \mathrm{m}$ in DM100model. Regions A, B, and C correspond to the temperature range defined below, within, and above the melting peak in the $c_{p}(T)$ curve. (b) $\Gamma(t)$ curves obtained from the MFH results and the line gives the heat variation within the interval $T_{\mathrm{i}}$ and $T_{\mathrm{F}}$. (c) $\Delta T(t)=T(t)-T_{0}$ curve measured for region $\mathrm{C}$ fitted with eq 7 and the temperature corrected for adiabatic conditions calculated using eq 8. (d) Estimated SPA values for regions A and $\mathrm{C}$, as well as the mean SPA $(\langle\mathrm{SPA}\rangle)$ estimated for region B. Similar curves for samples $x=0.19, x=0.12$, and $x=0.11$ in the same concentration are presented in $(\mathrm{e}-\mathrm{p})$.

with the reduction in the viscosity of $\mathrm{CBO}$ above the melting point.

A reproducible behavior was observed in the $T(t)$ curves after three successive heating cycles within $T_{0}<T \leq 301 \mathrm{~K}$, and subsequent cooling (Figure 6a, runs 1, 2, and 3 ) in the D5F1-model $\left(f=570 \mathrm{kHz}\right.$ and $\left.H_{0}=16 \mathrm{kA} / \mathrm{m}\right)$ for $\mathrm{CBO}$ containing 0.15 wt $\%$ of sample $x=0.19$. On the other hand, heating the samples above the melting region resulted in a clear nonreproducible data (runs 4 and 5 in Figure 6b). It can be noted that the $T(t)$ curve for run 4 is similar to run 3 up to $T$ $\sim 301 \mathrm{~K}$, whereas in run 5, deviations from run 3 are observed even below that temperature. We associate this change in the heating of the system with the agglomeration process of the $\mathrm{NP}$ in the melted $\mathrm{CBO}$, being less viscous, probably induced by the magnetic field gradient inside the coil and, to a lesser extent, with gravity. Different spatial configurations and NP aggregation degrees after successive runs could change the SPA, as observed in the analysis presented in Figure 5, and it could also lead to the observation of hysteresis behavior. In fact, the formation of large asymmetric agglomerates (non- 

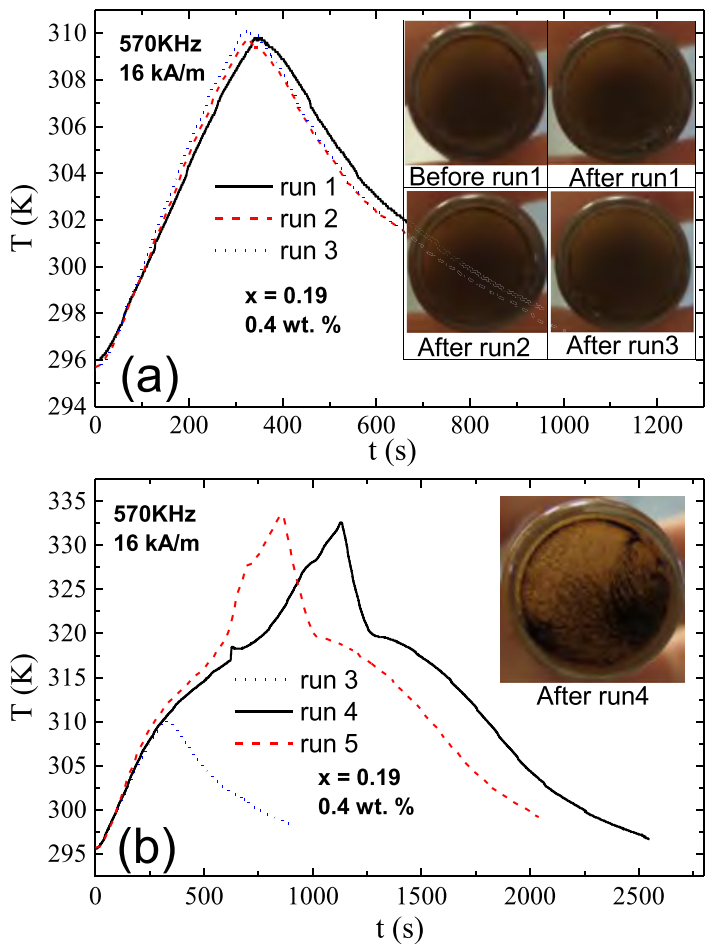

Figure 8. (a) MFH results for three subsequent runs performed in the D5-F1-model with $f=570 \mathrm{kHz}$ and $H_{0}=16 \mathrm{kA} / \mathrm{m}$ for paraffin containing 0.4 wt $\%$ of $x=0.11 \mathrm{NPs}$ from $T_{0}$ to a final temperature below $316 \mathrm{~K}$, followed by cooling to $T_{0}$. (b) Two subsequent runs (4 and 5) measured under the same experimental conditions and performed from $T_{0}$ to a final temperature above the melting temperature $\left(T>T_{\mathrm{F}}\right)$. Insets present the picture of the samples after the indicated run.

planar or bulk) is clearly observed by the naked eye after successive runs (see Figure $6 \mathrm{~b}$ ), which is not observed after successive runs when the heating temperature is kept below $301 \mathrm{~K}$ (see the inset of Figure 6a). The irreversible effect in MFH curves becomes reversible if the particles are dispersed again, for example, with ultrasound exposure, as shown in Figure $6 \mathrm{c}$ for three successive runs of the same sample measured in the D5-F1-model $\left(f=570 \mathrm{kHz}\right.$ and $H_{0}=16 \mathrm{kA} /$ $\mathrm{m}$ ) with intermediate exposure to ultrasound (about $20 \mathrm{~min}$ ).

Figure $7 \mathrm{a}-\mathrm{p}$ presents a similar analysis to that of Figure 5 for samples $x=0.21,0.19,0.12$, and 0.11 dispersed in paraffin at a concentration of $0.4 \mathrm{wt} \%$ with $H_{0}=16 \mathrm{kA} / \mathrm{m}$ and $f=570 \mathrm{kHz}$. Here, the quantity $\Delta Q_{\text {loss }}$ can be estimated from the equivalent curve from the cooling of paraffin until the temperature attains $T_{\mathrm{e}}$ (see Figure S9 in pg. S11 of Supporting Information). In general, the graphics present tendencies similar to those ones for the sample dispersed in $\mathrm{CBO}$, except for the higher temperature for the observation of increment in the heating rate and the effects of phase transition. In a similar way to the sample dispersed in CBO, Figure 8a shows the reversibility of hyperthermia results for sample $x=0.11$ dispersed in paraffin measured in the D5-F1-model $\left(f=570 \mathrm{kHz}\right.$ and $H_{0}=16 \mathrm{kA} /$ $\mathrm{m}$ ) when the maximum temperature attained is below $316 \mathrm{~K}$ (runs 1-3). Images taken (inset of Figure 8a) before and after each run evidence that no changes are observed in this temperature range. However, when the temperature overcomes the melting point (runs 4 and 5 in Figure $8 \mathrm{~b}$ ), NP agglomeration (see the inset of Figure $8 \mathrm{~b}$ ) and irreversibility are obtained, similarly to that observed for $\mathrm{CBO}$. Interestingly, the increment in the heating rate is not observed when the NPs are dispersed in toluene (see Figure S10 in pg. S12 of Supporting Information), without a phase transition in the temperature range of the MFH experiment, and agglomeration is observed in the beginning of the experiment.

Considering that the change in the MFH curves above the melting temperature can be correlated with this agglomeration process, some speculation can be made with respect to the agglomeration process. There is currently some debate about the effects of particle agglomeration on the heating mechanisms. While some works reported a reduction of the SPA due to the formation of bulk agglomerates, ${ }^{6,9}$ there is also experimental evidence that the formation of elongated agglomerates (e.g., needle-like, cylinder, or chain-like structures) increases the SPA compared to that of the welldispersed NPs. ${ }^{12-14}$ Recently, some theoretical models and experimental work showed that the actual effects of clustering could be either way, depending on the value of effective magnetic anisotropy and anisotropy energy barrier of NPs. ${ }^{35,36}$ Basic considerations from magnetostatics indicate that in the presence of a nonuniform magnetic field, the NPs tend to form elongated structures aligned with the magnetic field gradient. Probably, in our work, the reduction in the viscosity of $\mathrm{CBO}$ above the melting point allows the alignment of easy axis of NPs with the applied field at the same time that nonplanar structures, probably elongated ones, are induced by the combination of the magnetic field gradient inside the coil and, to a lesser extent, to gravity. Any real applicator solenoid (even in a perfectly constructed one) will contain a nonuniform magnetic field along the coil axis because of finite size effects. The formation of elongated structures after the first heating cycle, and the consequent increase in SPA in our case, is consistent with this explanation. According to this, the resulting heating curve will therefore depend on the magnetic field gradient (a characteristic of each MFH apparatus) and the NP concentration because of the interparticle interaction strength.

The low anisotropy barrier of the systems studied here could play an important role in the increment of the SPA after agglomeration. A system with a low anisotropy results in an increment in the SPA in the case of agglomeration by two reasons: first, the NPs tend to orient the easy axis with the applied field; second, in the case of formation of agglomerates with axial symmetry. ${ }^{37}$

The results presented in this work call for the attention of the complex heat-transfer process in MFH experiments from the NPs to the medium, where the thermodynamic characteristic of media should be taken into account to evaluate the efficiency of NP systems in MFH treatments when they are applied in a different environment. In particular, $\mathrm{CBO}$ and paraffin are used to emulate a high-viscosity environment and evaluate the efficiency of MFH when the Neel relaxation is the dominant mechanism, evidencing how the intrinsic transition of media could affect the heating in an MFH experiment. Several aspects such as the presence of latent heat in a transition, strong variation of specific heat with temperature, and the formation of NP agglomerates when the viscosity diminishes after melting are some of the aspects that should be evaluated in different media with a complex structure or complex thermal response to analyze the MFH results and therapy effectiveness. 


\section{CONCLUSIONS}

The SPA of $\mathrm{Zn}_{x} \mathrm{Fe}_{3-x} \mathrm{O}_{4}$ magnetic NPs dispersed in $\mathrm{CBO}$ and paraffin presents a complex behavior with temperature related to the melting of these media. Samples present distinct morphology and magnetic properties, specifically distinct anisotropy energy barrier. For all samples, the magnetic relaxation mechanism is dominated by Nèel relaxation, independent of the $\mathrm{CBO}$ or paraffin phase, below and above the melting points. The complex SPA behavior with temperature for this system is correlated with the nonlinear thermal response of viscosity and thermodynamic properties of the medium, including a phase transition in the temperature range of MFH experiment. The variation of $c_{p}$ of the medium with temperature, as well as the latent heat of melting, is important to analyze the SPA evolution of all samples. The heating rate strongly depends on the variation of these thermodynamic properties.

Despite the change in the viscosity of media with melting being not high enough to change the dominant relaxation mechanism, it allows the NP agglomeration induced by the applied field gradient of the MFH coil, which leads to changes in the SPA when the system is heated above the melting point. This effect in the SPA depends on the NP concentration, and it also results in a hysteresis in MFH experiments for subsequent runs when the melting temperature is attained.

Finally, this work highlights the importance of measuring the thermodynamic properties of media where the NPs would be applied, in order to evaluate the efficiency of MFH treatment, especially in complex media where different transitions could take place.

\section{ASSOCIATED CONTENT}

\section{SI Supporting Information}

The Supporting Information is available free of charge at https://pubs.acs.org/doi/10.1021/acs.jpcc.0c06843.

Compositional analysis by PIXE of the four samples; XRD measurements of sample $x=0.21$ as a representative of samples; saturation magnetization $\left(\mathrm{M}_{\mathrm{S}}\right)$ determined from magnetic measurements; obtaining the blocking-temperature distribution $f\left(T_{\mathrm{B}}\right)$ from magnetization measurements as a function of temperature; magnetization measurements of $\mathrm{CBO}$ and paraffin without NPs at $300 \mathrm{~K}$; MFH measurements of CBO and paraffin without NPs; changes in the physical aspects of $\mathrm{CBO}$ with temperature; cooling curve of $\mathrm{CBO}+\mathrm{NPs}$ heated in a hot plate up to a temperature above the melting point and its analysis with respect to quantity $\Gamma(t)$; cooling curve of paraffin with NPs heated in a hot plate up to a temperature above the melting point and its analysis with respect to quantity $\Gamma(t)$; and $\mathrm{MFH}$ of the four samples dispersed in toluene (PDF)

\section{AUTHOR INFORMATION}

\section{Corresponding Author}

Enio Lima, Jr. - Instituto de Nanociencia y Nanotecnología, CNEA-CONICET, Centro Atómico Bariloche, S. C. de Bariloche 8400, Argentina; (ㄱ) orcid.org/0000-0002-60284274; Email: lima@cab.cnea.gov.ar

\section{Authors}

Adriele A. de Almeida - Instituto de Nanociencia y Nanotecnología, CNEA-CONICET, Centro Atómico Bariloche, S. C. de Bariloche 8400, Argentina

Emilio De Biasi - Instituto de Nanociencia y Nanotecnología, CNEA-CONICET and Instituto Balseiro, CNEA-UNCuyo, Centro Atómico Bariloche, S. C. de Bariloche 8400, Argentina

Marcelo Vasquez Mansilla - Instituto de Nanociencia y Nanotecnología, CNEA-CONICET, Centro Atómico Bariloche, S. C. de Bariloche 8400, Argentina

Daniela P. Valdés - Instituto de Nanociencia y Nanotecnología, CNEA-CONICET and Instituto Balseiro, CNEA-UNCuyo, Centro Atómico Bariloche, S. C. de Bariloche 8400, Argentina; (ㅇ orcid.org/0000-0002-84411856

Horacio E. Troiani - Instituto Balseiro, CNEA-UNCuyo and Laboratorio de Caracterización de Materiales y Óxidos NoEstequiométricos, Gerencia de Investigación Aplicada, Centro Atómico Bariloche, S. C. de Bariloche 8400, Argentina; (1) orcid.org/0000-0002-2259-3252

Guillermina Urretavizcaya - Consejo Nacional de Investigaciones Científicas y Técnicas (CONICET) e Instituto Balseiro, Centro Atómico Bariloche, S. C. de Bariloche 8400, Argentina

Teobaldo E. Torres - Instituto de Nanociencia y Nanotecnología, CNEA-CONICET, Centro Atómico Bariloche, S. C. de Bariloche 8400, Argentina; (1) orcid.org/ 0000-0002-6116-9331

Luis M. Rodríguez - Instituto de Nanociencia y Nanotecnología, CNEA-CONICET, Centro Atómico Bariloche, S. C. de Bariloche 8400, Argentina

Daniel E. Fregenal - Centro Atómico Bariloche, San Carlos de Bariloche 8400, Argentina

Guillermo C. Bernardi - Instituto de Nanociencia y Nanotecnología, CNEA-CONICET, Centro Atómico Bariloche, S. C. de Bariloche 8400, Argentina

Elin L. Winkler - Instituto de Nanociencia y Nanotecnología, CNEA-CONICET and Instituto Balseiro, CNEA-UNCuyo, Centro Atómico Bariloche, S. C. de Bariloche 8400, Argentina; (1) orcid.org/0000-0002-9575-7879

Gerardo F. Goya - Departamento de Física de la Materia Condensada and Instituto de Nanociencia de Aragón, Universidad de Zaragoza, Zaragoza 50018, Spain; (1) orcid.org/0000-0003-1558-9279

Roberto D. Zysler - Instituto de Nanociencia y Nanotecnología, CNEA-CONICET and Instituto Balseiro, CNEA-UNCuyo, Centro Atómico Bariloche, S. C. de Bariloche 8400, Argentina

Complete contact information is available at: https://pubs.acs.org/10.1021/acs.jpcc.0c06843

\section{Notes}

The authors declare no competing financial interest.

\section{ACKNOWLEDGMENTS}

The authors acknowledge financial support of Argentinian governmental agency ANPCyT (project no. PICT-2016-0288 and PICT-2015-0883) and UNCuyo (project no.06/C527 and 06/C528). The authors gratefully acknowledge the EUCommission financial support under the project: H2020MSCA-RISE-2016 SPICOLOST project no. 734187. G.F.G. and T.E.T. also acknowledge the financial support from the 
Spanish Ministerio de Ciencia, Innovación y Universidades (project MAT2016-78201-P) and the Aragon Regional Government (DGA, project no. E26).

\section{REFERENCES}

(1) Rosensweig, R. E. Heating magnetic fluid with alternating magnetic field. J. Magn. Magn. Mater. 2002, 252, 370.

(2) Lima, E., Jr.; De Biasi, E.; Zysler, R. D.; Vasquez Mansilla, M.; L Mojica-Pisciotti, M.; Torres, T. E.; Pilar Calatayud, M.; Marquina, C.; Ibarra, M. R.; Goya, G. F. Relaxation time diagram for identifying heat generation mechanisms in magnetic fluid hyperthermia. J. Nanopart. Res. 2014, 16, 2791.

(3) Fabris, F.; Lima, E., Jr.; De Biasi, E.; Troiani, H. E.; Vásquez Mansilla, M.; Torres, T. E.; Fernández Pacheco, R.; Ibarra, M. R.; Goya, G. F.; Zysler, R. D.; et al. Controlling the dominant magnetic relaxation mechanisms for magnetic hyperthermia in bimagnetic core-shell nanoparticles. Nanoscale 2019, 11, 3164.

(4) Carrey, J.; Mehdaoui, B.; Respaud, M. Simple models for dynamic hysteresis loop calculations of magnetic single-domain nanoparticles: Application to magnetic hyperthermia optimization. J. Appl. Phys. 2011, 109, 083921.

(5) Torres, T. E.; Lima, E., Jr.; Calatayud, M. P.; Sanz, B.; Ibarra, A.; Fernández-Pacheco, R.; Mayoral, A.; Marquina, C.; Ibarra, M. R.; Goya, G. F. The relevance of brownian relaxation as power absorption mechanism in magnetic hyperthermia. Sci. Rep. 2019, 9, 3992.

(6) Lima, E., Jr.; De Biasi, E.; Mansilla, M. V.; Saleta, M. E.; Granada, M.; Troiani, H. E.; Effenberger, F. B.; Rossi, L. M.; Rechenberg, H. R.; Zysler, R. D. Heat generation in agglomerated ferrite nanoparticles in an alternating magnetic field. J. Phys. D: Appl. Phys. 2013, 46, 045002.

(7) Sanz, B.; Calatayud, M. P.; De Biasi, E.; Lima, E., Jr.; Vasquez Mansilla, M.; Zysler, R. D.; Ibarra, M. R.; Goya, G. F. In silico before in vivo: how to predict the heating efficiency of magnetic nanoparticles within the intracellular space. Sci. Rep. 2016, 6, 38733.

(8) Sadat, M. E.; Patel, R.; Sookoor, J.; Bud'ko, S. L.; Ewing, R. C.; Zhang, J.; Xu, H.; Wang, Y.; Pauletti, G. M.; Mast, D. B.; et al. Effect of spatial confinement on magnetic hyperthermia via dipolar interactions in $\mathrm{Fe} 3 \mathrm{O} 4$ nanoparticles for biomedical applications. Mater. Sci. Eng., C 2014, 42, 52.

(9) Branquinho, L. C.; Carrião, M. S.; Costa, A. S.; Zufelato, N.; Sousa, M. H.; Miotto, R.; Ivkov, R.; Bakuzis, A. F. Effect of magnetic dipolar interactions on nanoparticle heating efficiency: implications for cancer hyperthermia. Sci. Rep. 2013, 3, 2887.

(10) Coral, D. F.; Mendoza Zélis, P.; Marciello, M.; Morales, M. d. P.; Craievich, A.; Sánchez, F. H.; Fernández van Raap, M. B. Effect of nanoclustering and dipolar interactions in heat generation for magnetic hyperthermia. Langmuir 2016, 32, 1201.

(11) Hiergeist, R.; Andrä, W.; Buske, N.; Hergt, R.; Hilger, I.; Richter, U.; Kaiser, W. Application of magnetite ferrofluids for hyperthermia. J. Magn. Magn. Mater. 1999, 201, 420.

(12) Mehdaoui, B.; Tan, R. P.; Meffre, A.; Carrey, J.; Lachaize, S.; Chaudret, B.; Respaud, M. Increase of magnetic hyperthermia efficiency due to dipolar interactions in low-anisotropy magnetic nanoparticles: Theoretical and experimental results. Phys. Rev. B 2013, $87,174419$.

(13) Martinez-Boubeta, C.; Simeonidis, K.; Makridis, A.; Angelakeris, M.; Iglesias, O.; Guardia, P.; Cabot, A.; Yedra, L.; Estradé, S.; Peiró, F.; et al. Learning from nature to improve the heat generation of iron-oxide nanoparticles for magnetic hyperthermia applications. Sci. Rep. 2013, 3, 1652.

(14) Serantes, D.; Simeonidis, K.; Angelakeris, M.; ChubykaloFesenko, O.; Marciello, M.; del Puerto Morales, M.; Baldomir, D.; Martinez-Boubeta, C. Multiplying magnetic hyperthermia response by nanoparticle assembling. J. Phys. Chem. C 2014, 118, 5927.

(15) Vakhshouri, A. R. Paraffin as phase change material. In Paraffin, An Overview; Soliman, F. S., Ed.; IntechOpen: London, 2020; Chapter 5 .
(16) Jesumathy, P. S. Latent heat thermal energy storage system. In Paraffin, An Overview; Soliman, F. S., Ed.; IntechOpen: London, 2020; Chapter 4.

(17) Mehta, B. M. Butter, butter oil and ghee. In Gourmet and Health-Promoting Specialty Oils; Moreau, R. A., Kamal-Eldin, A., Eds.; AOCS Press-Urbana: Illinois, 2009; Chapter 21, p 527.

(18) Rønholt, S.; Buldo, P.; Mortensen, K.; Andersen, U.; Knudsen, J. C.; Wiking, L. The effect of butter grains on physical properties of butter-like emulsions. J. Dairy Sci. 2014, 97, 1929.

(19) Changade, S. P.; Tambat, R. V.; Kanoje, R. R. Physical properties of ghee prepared from high acidic milk-II. J. Dairy. Foods Home Sci. 2006, 25, 101.

(20) Devl, A.; Khatkar, B. Thermo-physical properties of fats and oils. Int. J. Res. Eng. Technol. Res. 2017, 7, 45.

(21) Duhan, N.; Sahu, J. K.; Naik, S. N. Temperature dependent steady and dynamic oscillatory shear rheological characteristics of indian cow milk (Desi) ghee. J. Food Sci. Technol. 2018, 55, 4059.

(22) Watson, E. L. Thermal properties of butter. Can. Agric. Eng. 1975, 17, 68.

(23) Tomaszewska-Gras, J. Melting and crystallization DSC profiles of milk fat depending on selected factors. J. Therm. Anal. Calorim. 2013, 113, 199.

(24) Lohr, J.; de Almeida, A. A.; Moreno, M. S.; Troiani, H.; Goya, G. F.; Torres Molina, T. E.; Fernández-Pacheco, R.; Winkler, E. L.; Vasquez Mansilla, M.; Cohen, R.; et al. Effects of $\mathrm{Zn}$ substitution in the magnetic and morphological properties of Fe-oxide-based coreshell nanoparticles produced in a single chemical synthesis. J. Phys. Chem. C 2019, 123, 1444.

(25) Johansson, S. A. E.; Campbell, J. L.; Malmqvist, K. G. ParticleInduced X-ray Emission Spectrometry (PIXE); Winefordner, J. D., Ed.; John Wiley \& Sons, 1995.

(26) Campbell, J. L.; Boyd, N. I.; Grassi, N.; Bonnick, P.; Maxwell, J. A. The Guelph PIXE software package IV. Nucl. Instrum. Methods Phys. Res., Sect. B 2010, 268, 3356.

(27) Lavorato, G. C.; Lima, E., Jr.; Lima, D., Jr.; Fiorani, D.; Troiani, H. E.; Zysler, R. D.; Winkler, E. L. Size effects in bimagnetic CoO/ CoFe2O4 core/shell nanoparticles. Nanotechnology 2014, 25, 355704.

(28) Mamiya, H.; Ohnuma, M.; Nakatani, I.; Furubayashim, T. Extraction of blocking temperature distribution from zero-field-cooled and field-cooled magnetization curves. IEEE Trans. Magn. 2005, 41, 3394.

(29) Balaev, D. A.; Semenov, S. V.; Dubrovskiy, A. A.; Yakushkin, S. S.; Kirillov, V. L.; Martyanov, O. N. Superparamegntic blocking in an ensemble of magnetite nanoparticles upon interparticle interactions. $J$. Magn. Magn. Mater. 2017, 440, 199.

(30) Usov, N. A. Numerical simulation of field-cooled and zero fieldcooled processes for assembly of superparamagnetic nanoparticles with uniaxial anisotropy. J. Appl. Phys. 2011, 109, 023913.

(31) Livesey, K. L.; Ruta, S.; Anderson, N. R.; Baldomir, D.; Chantrell, R. W.; Serantes, D. Beyond the blocking model to fit nanoparticle ZFC/FC magnetization curves. Sci. Rep. 2018, 8, 11166. (32) Muscas, G.; Concas, G.; Laureti, S.; Testa, A. M.; Mathieu, R.; De Toro, J. A.; Cannas, C.; Musinu, A.; Novak, M. A.; Sangregorio, C.; et al. The interplay between single particle anisotropy and interparticle interactions in ensembles of magnetic nanoparticles. Phys. Chem. Chem. Phys. 2018, 20, 28634.

(33) Höhne, G. W. H.; Hemminger, W. F.; Flammersheim, H.-J. Differential Scanning Calorimetry; Springer: Berlin, 2003.

(34) Natividad, E.; Castro, M.; Mediano, A. Adiabatic vs. nonadiabatic determination of specific absorption rate of ferrofluids. $J$. Magn. Magn. Mater. 2009, 321, 1497.

(35) Abu-Bakr, A. F.; Zubarev, A. Y. On the theory of magnetic hyperthermia: clusterization of nanoparticles. Philos. Trans. R. Soc., A 2020, 378, 20190251.

(36) Myrovali, E.; Maniotis, N.; Makridis, A.; Terzopoulou, A.; Ntomprougkidis, V.; Simeonidis, K.; Sakellari, D.; Kalogirou, O.; Samaras, T.; Salikhov, R.; et al. Arrangement at the nanoscale: effect on magnetic particle hyperthermia. Sci. Rep. 2016, 6, 37934. 
(37) Valdés, D. P.; Lima, E., Jr.; Zysler, R. D.; De Biasi, E. Modeling the magnetic-hyperthermia response of linear chains of nanoparticles with low anisotropy: a key to improving specific power absorption.

Phys. Rev. Appl. 2020, 14, 014023. 